\title{
HIGH ENERGY PHYSICS
}

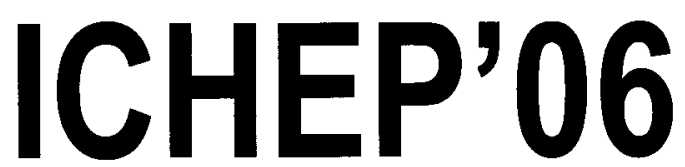

Volume I

Proceedings of the 33rd International Conference 
This page intentionally left blank 

Published by

World Scientific Publishing Co. Pte. Ltd.

5 Toh Tuck Link, Singapore 596224

USA office: 27 Warren Street, Suite 401-402, Hackensack, NJ 07601

UK office: 57 Shelton Street, Covent Garden, London WC2H 9HE

\section{British Library Cataloguing-in-Publication Data}

A catalogue record for this book is available from the British Library.

\section{HIGH ENERGY PHYSICS ICHEP 2006}

Proceedings of the 33rd International Conference

Copyright $\odot 2007$ by World Scientific Publishing Co. Pte. Ltd.

All rights reserved. This book, or parts thereof, may not be reproduced in any form or by any means, electronic or mechanical, including photocopying, recording or any information storage and retrieval system now known or to be invented, without written permission from the Publisher.

For photocopying of material in this volume, please pay a copying fee through the Copyright Clearance Center, Inc., 222 Rosewood Drive, Danvers, MA 01923, USA. In this case permission to photocopy is not required from the publisher.

$\begin{array}{lll}\text { ISBN-13 } & 978-981-270-994-3 & \text { (Vol. 1) } \\ \text { ISBN-10 } 981-270-994-0 & \text { (Vol. 1) } \\ \text { ISBN-13 } 978-981-270-995-0 & \text { (Vol. 2) } \\ \text { ISBN-10 } 981-270-995-9 & \text { (Vol. 2) } \\ \text { ISBN-13 } 978-981-270-385-9 & \text { (Set) } \\ \text { ISBN-10 } 981-270-385-3 & \text { (Set) }\end{array}$




\section{PREFACE}

The Rochester conference ICHEP'06, the thirty third in this series, was held on 26 July - 2 August, 2006 in the main building of the Russian Academy of Sciences in Moscow.

About a thousand physicists from 53 countries took part in the conference. The extensive scientific program consisted of 27 plenary reports and 365 original presentations at 14 parallel sessions. In addition, 95 poster presentations were displayed. For six days, the conference participants discussed urgent issues of astrophysics and cosmology, neutrino physics, lattice and quantum field theory, heavy-ion collisions and quark-gluon matter, hard and soft processes in quantum chromodynamics, physics of electroweak interactions, rare decays of elementary particles, spectroscopy and exotic states of hadrons, heavy quark physics, theoretical scenarios and experimental searches beyond the Standard Model, creation of a unified system GRID for data processing, new accelerators and particle detectors, mathematical aspects of quantum field theory and string theory.

On the theory side, the progress in the so-called "practical theory" is evident, primarily in sophisticated calculations in quantum chromodynamics. Splendid harmony of analytical calculations with the results obtained on the lattice with account of dynamics of quarks is a remarkable achievement. Considerable emphasis in theoretical discussions was placed on the concept and use of gravity/gauge duality, the framework generalizing the renown AdS/CFT correspondence. This duality is a conjectured relationship between confining gauge theories in four dimensions, on the one hand, and gravity and string theory in five and more dimensions, on the other.

On experimental side, numerous candidates for exotic hadronic states, both with light quarks only and with heavy quarks and/or gluons, were confirmed or newly reported. These exotic states are yet to be given theoretical interpretation, within either gravity/gauge duality or more traditional approaches.

A relatively novel area of QCD - properties of matter at high temperatures and particle densities - is under intense study at the Relativistic Heavy Ion Collider in Brookhaven.

With the B-factories and Tevatron in operation, the conference witnessed impressive progress in flavor physics, including B-meson decays, processes with $\mathrm{CP}$-violation.

Precision measurements of the masses of the heaviest known particles are still an important aspect of experimental high energy physics. New results in this area presented at the conference were based mainly on the data from the CDF and DO collaborations at the Tevatron.

Considerable attention at this conference was paid to the search for new physics. Numerous possible properties beyond the Standard Model, such as supersymmetry, extra spacetime dimensions, effective contact interactions in quark and lepton sectors, additional heavy gauge bosons, excited states of quarks and leptons, leptoquarks, etc., are more constrained than before. Yet the majority of the community is confident that new physics is within the reach of LHC, and, indeed, more theoretical scenarios for $\mathrm{TeV}$-scale physics beyond the Standard Model were presented at the conference. 
More generally, the atmosphere at the conference was largely determined by the fact that it was the last pre-LHC Rochester meeting.

Properties of neutrinos continue to be among the top issues. The first data reported at this conference by the new player - MINOS collaboration -.- support the pattern of the oscillations of muon neutrinos observed by Super-K and K2K.

Astroparticle physics is another area of continuing interest. The new measurement of the neutron lifetime contributes considerably to the calculation of the abundance of primordial Helium-4 in the Universe. Techniques for the direct and indirect detection of dark matter particles are rapidly developing, with indications for positive signals from DAMA and EGRET still persisting.

Traditionally, the Rochester conference discusses future accelerators for high energy physics and new developments in particle detection, and receive reports from the International Committee for Future Accelerators (ICFA) and IUPAP Commission on Particles and Fields (C11). This was particularly timely in Moscow in view of the upcoming LHC. At present, the world scientific community is widely discussing a new mega-project of the 21st century - the ILC. Together with the LHC at CERN, the new machine will be a unique tool for studying fundamental properties of matter and the Universe.

In his concluding report V. Rubakov (INR Moscow) summarized the discussions at the conference with emphasis on the current confusion of (some) theorists regarding new physics and on the impact of LHC on the entire field and beyond. Hopefully, with the first results from $\mathrm{LHC}$, at least some of the numerous questions raised in Moscow will be answered at the next Rochester conference - 2008 in Philadelphia.

The participants and guests of the conference took part in an extensive, traditionally hospitable cultural program including excursions around Moscow, the Moscow region, St.Petersburg and other Russian cities after the conference.

We wish to thank the members of the Organizing Committee, the conveners of parallel sessions who worked hard to make the Conference a success. Special thanks are due to members of the International Advisory Program Committee for setting-up the scientific directions at an early stage of planning. We would also like to take this opportunity to express our sincere gratitude to all those who gave us financial and/or logistic support: International Union of Pure and Applied Physics, the Russian Academy of Sciences, the Moscow State University, the Joint Institute for Nuclear Research, the RF Federal Agency of Science and Innovation.

The ICHEP'06 conference was jointly organized by the Joint Institute for Nuclear Research with the International Intergovernmental Organization, the Russian Academy of Sciences, the Russian Federation Ministry of Education and Science, the RF Federal Agency on Science and Innovation, the RF Federal Agency on Atomic Energy and the Lomonosov Moscow State University under the leading role of IUPAP.
V. A. Matveev (INR RAS, Moscow)
A. N. Skrinsky (BINP, Novosibirsk)
V. I. Savrin (SINP MSU, Moscow)
A. N. Sissakian (JINR) 


\title{
Organizers
}

Russian Academy of Sciences

Ministry of Education and Science of the Russian Federation

Federal Agency of Science and Innovations of the Russian Federation

Federal Atomic Energy Agency of the Russian Federation

Moscow State University

Joint Institute for Nuclear Research (Dubna)

\begin{abstract}
Co-chairmen: V.A. Matveev (RAS, Moscow) \& A.N. Skrinsky (RAS, Moscow) Vice-chairmen: V.I. Savrin (MSU, Moscow) \& A.N. Sissakian (JINR, Dubna)
\end{abstract}

\section{International Advisory and Program Committee}

\author{
G. Altarelli (CERN) \\ S. Banerjee (TATA) \\ N. Brambilla (Milano) \\ R. Cashmore (Oxford) \\ H. Chen (IHEP, Beijing) \\ E. Coccia (Gran Sasso) \\ R. Eichler (PSI) \\ J. Engelen (CERN) \\ L. Foa (Pisa) \\ A. Fursenko (Moscow)
}

D. Gross (UCSB)

W.F. Henning (GSI)

V.G. Kadyshevsky (JINR)

A.A. Logunov (Protvino)

L. Maiani (Roma)

G. Mikenberg (Weizmann)

M. Neubert (Cornell)

L.B. Okun (ITEP)

Yu.S. Osipov (Moscow)

A.V. Radyushkin (JLAB)
L. Randall (Harvard)

A. Roodman (SLAC)

A.Yu. Rumyantsev (Moscow)

V.A. Sadovnichiy (MSU)

M. Spiro (IN2P3)

A.N. Tavkhelidze (JINR)

Y. Totsuka (KEK)

A. Wagner (DESY)

A.K. Wroblewsky (Warsaw)

Young-Kee Kim (Fermilab)

\section{Organizing Committee}

$\begin{array}{lll}\text { E.E. Antipenko (RosAtom) } & \text { A.V. Klimenko (RosNauka) } & \text { O.O. Patarakin (RosAtom) } \\ \text { V.V. Belokurov (MSU) } & \text { O.L. Kodolova (MSU) } & \text { A.A. Petrukhin (MEPhI) } \\ \text { L.B. Bezrukov (INR RAS) } & \text { G.A. Kozlov (JINR) } & \text { V.A. Rubakov (INR RAS) } \\ \text { P.V. Bogdanov (RosAtom) } & \text { Yu.F. Kozlov (RosNauka) } & \text { A.A. Slavnov (MI RAS) } \\ \text { M.V. Danilov (ITEP) } & \text { A.N. Lebedev (LPI RAS) } & \text { Yu.A. Tikhonov (BINP) } \\ \text { I.M. Dremin (LPI RAS) } & \text { O.Yu. Lukina (MSU) } & \text { N.E. Tyurin (IHEP) } \\ \text { V.G. Drozhenko (RosNauka) } & \text { V.A. Nazarenko (St.Petersburg) } & \text { A.A.Vorobijov (St.Petersburg) } \\ \text { A.B. Kaidalov (ITEP) } & \text { M.I. Panasyuk (MSU) } & \text { A.M. Zaitsev (IHEP) }\end{array}$

Scientific Secretary: G.A. Kozlov

Joint Institute for Nuclear Research

\section{Supported by}

International Union of Pure and Applied Physics (IUPAP)

Russian Academy of Sciences

Joint Institute for Nuclear Research (Dubna)

Russian Foundation for Basic Research

Moscow State University

Federal Agency of Science and Innovations of the Russian Federation

Comstar-UTS 
Photos compilation by B. Starchenko

Photos by Yu. Tumanov

Symposium logotype design by Yu. Meshenkov 


\section{CONTENTS}

\section{VOLUME I}

Preface

$\mathrm{v}$

\section{PLENARY SESSION}

Status of $\left|V_{u b}\right|,\left|V_{c b}\right|$ and Their Relative Phase. 3

R. V. Kowalewski

Charm and Charmonium: Spectroscopy, Production and Decays 13

P. N. Pakhlov

Rare B and $\tau$ Decays and Searches for New Physics 23 R. J. Barlow

QCD Review 33

G. Marchesini

Progress in Lattice QCD

G. Schierholz

New BES and CLEO Results

W. $L i$

Heavy Ion Theory: QCD and Matter in Extremis 64 X.-N. Wang

Heavy Hadron Spectroscopy A. Vairo

Light Quark Spectroscopy A. Zaitsev

Beyond the Standard Model 92 D. I. Kazakov

Experimental Results on Searches Beyond the Standard Model 103 E. Gallo

Electroweak Physics

D. R. Wood

Hot Topics from the Tevatron

D. Glenzinski 
New Results from Accelerator Neutrino Experiments . . . . . . . . . . . . . . 134

R. A. Rameika

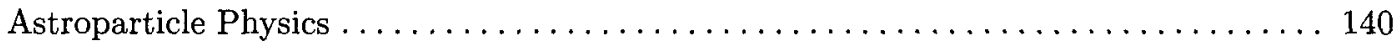

A. Bettini

Strings for Quantumchromodynamics

$V$. Schomerus

Preparing for Physics at the LHC

F. Gianotti

Accelerator Prospects for High Energy Physics.

A. N. Skrinsky

Conclusions and Outlook

V. A. Rubakov

\section{SESSION I. PARTICLE ASTROPHYSICS \& COSMOLOGY}

Cosmology and Colliders

R. Arnowitt, A. Arusano, B. Dutta, T. Kamon, N. Kolev, P. Simeon,

D. Toback and P. Wagner

Neutrinos, Dark Matter and Baryon Asymmetry of the Universe

M. Shaposhnikov

LIGO and VIRGO: Large Interferometers Searching for Gravitational Waves

G. Losurdo (for the LIGO Scientific Collaboration and the VIRGO Collaboration)

DAMA at GRAN SASSO: Results and Perspectives

R. Bernabei, P. Belli, F. Montecchia, F. Nozzoli, F. Cappella, A. Incicchitti,

D. Prosperi, R. Cerulli, C. J. Dai, H. L. He, H. H. Kuang, J. M. Ma,

$X$. D. Sheng and Z. P. Ye

Observation of Vacuum Birefringence Induced by a Transverse Magnetic Field

E. Zavattini, G. Zavattini, P. Temnikov, G. Ruoso, G. Raiteri, E. Polacco,

E. Milotti, M. Karuza, U. Gastaldi, G. Di Domenico, F. Della Valle,

R. Cimino, S. Carusotto, G. Cantatore and M. Bregant

Search for Dark Matter with Space Experiments

A. Morselli

Indirect Search for Dark Matter with AMS in Positrons, Gamma and

Antiprotons Channels

D. Casadei

Launch and Performance of the PAMELA Experiment

P. Picozza and M. Casolino (on behalf of PAMELA Collaboration) 


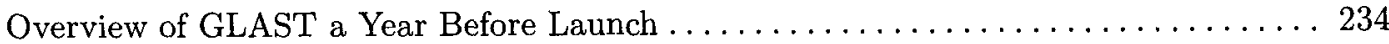

E. D. Bloom (representing the GLAST LAT Collaboration)

Galactic Model Uncertainties in Indirect Dark Matter Search . . . . . . . . . . . 238

W. De Boer, I. Gebauer, C. Sander, V. Zhukov and M. Weber

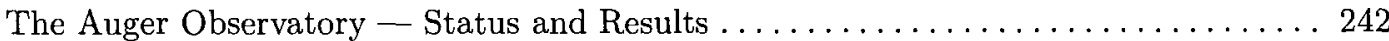

G. Matthiae (for the AUGER Collaboration)

Very High Energy Gamma-Ray Astronomy and Cosmic Ray Physics with

ARGO-YBJ . . . . . . . . . . . . . . . . . . . . . . . . . . . . . . . . . 247

I. De Mitri (on behalf of the ARGO-YBJ Collaboration)

Neutrino Astronomy and Astrophysics with IceCube and AMANDA . . . . . . 251

A. Olivas (for the ICECUBE Collaboration)

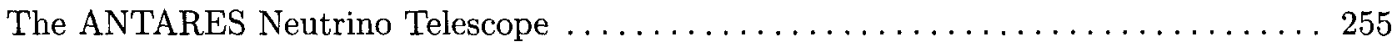

M. Circella (on behalf of the ANTARES Collaboration)

Probing Supernova Shock Waves and Matter Density Fluctuations by

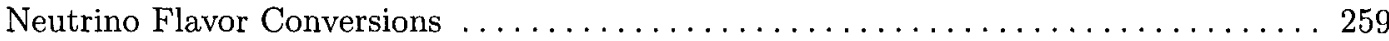

G. L. Fogli, E. Lisi, A. Mirizzi and D. Montanino

\section{SESSION II. NEUTRINO PHYSICS}

First MINOS Results from the NuMI Beam 265

G. F. Pearce (for the MINOS Collaboration)

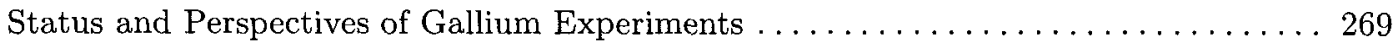

$V$. Gorbachev

Results from the HARP Experiment. . . . . . . . . . . . . . . . . . . . . . . 272

L. Howlett (on behalf of the HARP Collaboration)

NEMO-3 Double Beta Decay Experiment: Lastest Results . . . . . . . . . . . . . . . . . 276

A. S. Barabash (on behalf of the NEMO Collaboration)

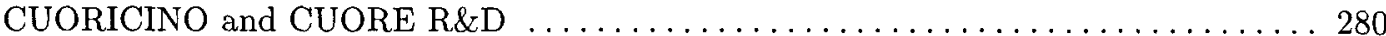

S. Capelli, R. Ardito, F. Alessandria, C. Arnaboldi, C. Brofferio, L. Carbone,

M. Clemenza, O. Cremonesi, E. Fiorini, A. Giuliani, G. Maier, C. Nones,

A. Nucciotti, M. Pavan, M. Pedretti, G. Pessina, S. Pirro, E. Previtali,

S. Sangiorgio, M. Sisti, L. Torres, L. Zanotti, F. Avignone, I. Bandac,

R. Creswick, H. Farach, C. Martinez, C. Rosenfeld, M. Balata, C. Bucci,

S. Nisi, C. Zarra, M. Barucci, L. Risegari, G. Ventura, J. W. Beeman,

M. Dolinski, S. J. Freedmann, D. Gutierrez, E. E. Haller, K. M. Heeger,

Yu. G. Kolomenski, R. Maruyama, E. B. Norman, A. R. Smith, N. Xu,

S. Cebrian, P. Gorla, I. G. Irastorza, S. Cuneo, S. Di Domizio, A. Giachero,

E. Guardincerri, M. Olcese, P. Ottonello, M. Pallavicini, V. Palmieri,

F. Bellini, C. Cosmelli, I. Dafinei, M. Diemoz, F. Ferroni, C. Gargiullo,

E. Longo, S. Morganti and M. Vignati 
A Global Analysis of Neutrino Oscillations .......................... 284

G. L. Fogli, E. Lisi, A. Marrone, A. Melchiorri, A. Palazzo, P. Serra, J. Silk and A. Slosar

Can We Learn Something More on Oscillations from Atmospheric Neutrinos?....... 288 T. Schwetz

Physics with India-Based Neutrino Observatory (INO) ................... 292 A. Dighe (for INO Collaboration)

$\mathrm{TeV}$ Leptogenesis in the Minimal Seesaw Model........................ 296 Z.-Z. Xing and S. Zhou

Status of the T2K Experiment.................................... 300 Yu. G. Kudenko (for the T2K Collaboration)

Minimal Lepton Flavour Violation and Leptogenesis ...................... 304 V. Porretti

From Unified Theories to Precision Neutrino Experiments .................. 308 S. Antusch

Realizations of $\mu-\tau$ Interchange Symmetry 312 W. Grimus

$A_{4}$ Symmetry Breaking Scheme for Understanding Quark and Lepton Mixing Angles...

R. R. Volkas

MSGUT: The Next Avtar ...................................... 320

C. S. Aulakh

eV Seesaw with Four Generations and $3+3$ Scenario

A. Soddu

OPERA - Status of the Experiment 328

Yu. Gornushkin

Status of Double Chooz Experiment............................... 332

V. V. Sinev

Measuring $\sin ^{2} 2 \theta_{13}$ with the Daya Bay Nuclear Power Reactors. Y.-F. Wang (for the Daya Bay Collaboration)

The KATRIN Experiment

M. Beck (for the KATRIN Collaboration)

Research Program Towards Observation of Neutrino-Nucleus Coherent Scattering ... 344 H. T. Wong, H. B. Li, S. K. Lin, S. T. Lin, D. He, J. Li, X. Li, Q. Yue, Z. Y. Zhou and S. K. Kim 


\section{SESSION III. LATTICE CALCULATIONS \& COMPUTATIONAL QUANTUM FIELD THEORY}

Impact of Lattice QCD Calculations on Unitarity Triangle Fits 351

M. Bona (representing the UTfit Collaboration)

Lattice QCD with Light Twisted Quarks: First Results 355

A. Shindler (on behalf of the European Twisted Mass Collaboration (ETMC))

Analytic Estimate of the Order Parameter for Magnetic Charge Condensation in QCD.

\section{A. Di Giacomo}

Implications of the Lattice Strings

V. I. Zakharov

Sonification of Monopoles and Chaos in QCD . . .

A. De Campo, N. Hörmann, H. Markum, W. Plessas and K. Vogt

Gribov Copy Problem in Lattice Gauge Theory Simulations 371

V. G. Bornyakov

\section{SESSION IV. HEAVY ION COLLISIONS \& QUARK MATTER}

Bulk Phenomena in High-Energy Nuclear Collisions at RHIC . .

N. $X u$

Global Observables at RHIC

A. Milou

Equation of State Near the QCD Critical Point

L. B. Bravina, I. C. Arsene, M. S. Nilsson, K. Tywoniuk and E. E. Zabrodin

Collective Effects in a Medium and a Model of Compound Flow in Relativistic

Heavy Ion Collisions.

V. A. Okorokov

Hard Processes in Heavy Ion Collisions N. Armesto

Debye Screening in the Hot Non-Abelian Plasma

N. O. Agasian and Yu. A. Simonov

Simulation of Jet Quenching in Heavy Ion Collisions 401

I. P. Lokhtin and A. M. Snigirev

Phases of Strongly Interacting Matter in the BRAHMS Experiment 405

P. Staszel (for the BRAHMS Collaboration) 


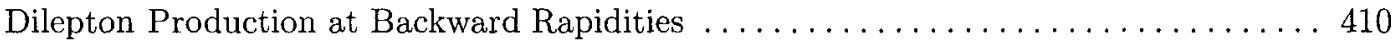

M. A. Betemps, M. B. Gay Ducati and E. G. de Oliveira

Ultra-Peripheral Heavy Ion Collisions at STAR

S. L. Timoshenko (for the STAR Collaboration)

Femtoscopic Correlations in Heavy Ion Collisions

R. Lednicky

A Search for a Mixed Quark-Hadron Phase of QCD Matter

A. N. Sissakian, A. S. Sorin and V. D. Toneev

Crossover, Fluctuations and Anderson Transition in Quark Matter Formation

B. Kerbikov

$\mathrm{J} / \psi$ Production in $158 \mathrm{GeV}$ Indium-Indium Collisions

H. K. Wöhri et al. (for the NA60 Collaboration)

Heavy Ion Physics with the CMS Detector $\ldots \ldots \ldots \ldots \ldots \ldots \ldots \ldots \ldots \ldots \ldots \ldots$

L. I. Sarycheva (for CMS Collaboration)

Observation of Quarkonia with the CMS Detector at LHC

O. Kodolova (on behalf of CMS Collaboration)

Scale Dependent Analysis Approach for STAR Events

O. V. Rogachevsky (for STAR Collaboration)

\section{SESSION V. QCD HARD INTERACTIONS}

Review of $\alpha_{\mathrm{S}}$ Measurements...

S. Kluth

Measurement of $\alpha_{\mathrm{S}}$ in $\mathrm{e}^{+} \mathrm{e}^{-}$Collisions at LEP and JADE

J. Schieck

Jets at LHC

I. Vivarelli (on behalf of the ATLAS Collaboration)

Inclusive Jet and Hadron Production in DIS at NLO

A. Daleo and R. Sassot

Inclusive Jet Production from the Tevatron

A. Kupčo (on behalf of the $C D F$ and DØ Collaborations)

$\mathrm{W}$ and $\mathrm{Z}$ Production at the Tevatron

M. P. Sanders (for the $D \varnothing$ and CDF Collaborations)

$\mathrm{W} / \mathrm{Z}+$ jets and $\mathrm{Z} p_{\mathrm{T}}$ Measurements at Tevatron

$Y$. S. Chung (on behalf of the CDF and DØ Collaborations) 
Photon Production at the Tevatron $\ldots \ldots \ldots \ldots \ldots \ldots \ldots \ldots \ldots \ldots \ldots \ldots \ldots$

C. Schwanenberger (on behalf of the CDF and DØ Collaborations)

Results from Two-Photon Collisions at LEP $\ldots \ldots \ldots \ldots \ldots \ldots \ldots \ldots \ldots \ldots \ldots \ldots$

V. P. Andreev

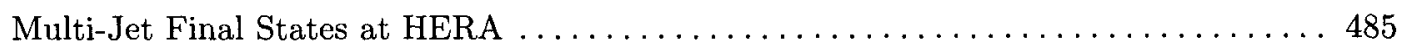

T. Gosau (for the ZEUS Collaboration)

Prompt Photons and Particle Momentum Distributions at HERA . . . . . . . . . 489 N. Gogitidze

Towards Precision Determination of Unintegrated Parton Density Functions 493 H. Jung, A. V. Kotikov, A. V. Lipatov and N. P. Zotov

Parton Distribution Functions and Jet Cross Sections at HERA

E. Tassi (on behalf of the H1 and ZEUS Collaborations)

Universal Anomalous Dimension and Pomeron in the $\mathcal{N}=4$ Supersymmetric

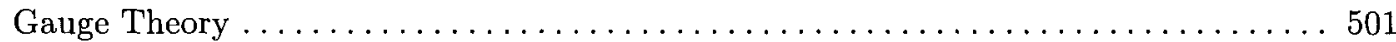

A. V. Kotikov, L. N. Lipatov, A. I. Onishchenko and V. N. Velizhanin

$\mathrm{QED} \otimes \mathrm{QCD}$ Exponentiation: Shower/ME Matching and IR-Improved DGLAP

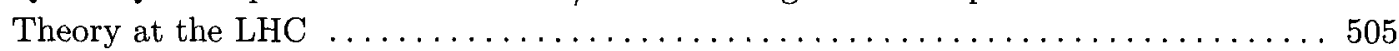

B. F. L. Ward and S. A. Yost

Gluon Polarisation Measurement at COMPASS ..................... 509

O. Kouznetsov (on behalf of COMPASS Collaboration)

New Results from HERMES . . . . . . . . . . . . . . . . . . . . . . 513

V. A. Korotkov (on behalf of the HERMES Collaboration)

Initial State Radiation (ISR) Study at BABAR and the Application to the

R Measurement and Hadron Spectroscopy . . . . . . . . . . . . . . . . . . . . . 517

E. P. Solodov (representing the BABAR Collaboration)

Four-Loop Tadpoles and Precision Calculations in Perturbative QCD . . . . . . . 521 Johann H. Kühn

Studies of Fragmentation and Colour Reconnection at LEP

P. Azzurri

Charm and Beauty Photoproduction at HERA M. Zambrana

Heavy Flavor Production at the Tevatron $K$. Hatakeyama (for the CDF and DØ Collaborations) 


\section{SESSION VI. QCD SOFT INTERACTIONS}

First Measurement of the $\pi^{+} \pi^{-}$Atom Lifetime . . . . . . . . . . . . . . . . . . 539

L. Tauscher (for the DIRAC Collaboration)

Measurements of $K_{e 4}$ and $K^{ \pm} \rightarrow \pi^{0} \pi^{0} \pi^{ \pm}$Decays $\ldots \ldots \ldots \ldots \ldots \ldots \ldots \ldots \ldots$

L. Masetti (for the NA48/2 Collaboration)

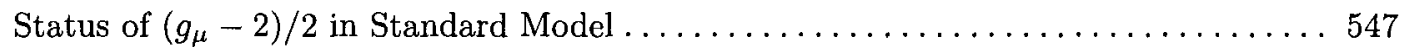

S. Eidelman

Hadronic Corrections to Muon g-2 Within Instanton Model

A. E. Dorokhov

Measurements of Form Factors with the BABAR Experiment

S. Z. Li (for the BABAR Collaboration)

Mini Review on Saturation and Recent Developments

C. Marquet

Infrared Freezing of Euclidean QCD Observables 563

C. J. Maxwell

Jet Fragmentation Studies at CDF 567

L. Pinera (on behalf of the CDF Collaboration)

Leading Systems, Colour Reconnection and Anomalous Soft Photons

F. Mandl

Particle Production and Fragmentation Results from ZEUS

A. Galas (on behalf of ZEUS Collaboration)

Studying the Underlying Event at CDF .

R. D. Field

Inclusive Jet Production from the Tevatron

A. Kupčo (on behalf of the CDF and DØ Collaborations)

Diffractive DIS Cross Sections and Parton Distributions F.-P. Schilling (for the H1 Collaboration)

QCD Fits to Diffraction

S. Sapeta

Diffractive Dijets and Open Charm Production at HERA

$M$. Kapishin (for the H1 and ZEUS Collaborations)

Leading Baryon Production at HERA

$K$. Borras (on behalf of the ZEUS and H1 Collaborations) 
DVCS and Vector Meson Production at HERA

$K$. Krüger (for the H1 and ZEUS Collaborations)

Diffractive Higgs Production

J. R. Forshaw

Diffraction at the Tevatron

A. Hamilton (on behalf of the CDF and DØ Collaborations)

Underlying Events and Diffraction Studies at the LHC . P. Bartalini

Forward Proton Detectors at High Luminosity at the LHC M. Grothe

Coupling Constants and High-Energy Scattering V. A. Petrov

Investigation of Multiple Production of Hadrons in Antiproton-Proton and Proton-Proton Interactions at 22.4, 32 and $69 \mathrm{GeV} / \mathrm{c}$

E. G. Boos, N. S. Pokrovsky, V. V. Samoilov, T. Temiraliev,

R. A. Tursunov and B. O. Zhautykov

Single Spin Asymmetries in Inclusive $\pi^{0}$ Production in $p+p$ and $\pi^{-}+p$

Interactions at $40-70 \mathrm{GeV}$

V. V. Mochalov, A. M. Davidenko, A. A. Derevschikov, Y. M. Goncharenko,

V. Y. Khodyrev, V. I. Kravtsov, Y. A. Matulenko, Y. M. Melnick,

A. P. Meschanin, N. G. Minaev, D. A. Morozov, L. V. Nogach,

S. B. Nurushev, L. F. Prudkoglyad, P. A. Semenov, L. F. Soloviev,

A. N. Vasiliev, A. E. Yakutin, N. L. Bazhanov, N. S. Borisov,

A. N. Fedorov, V. G. Koloimiets, A. B. Lazarev, A. B. Neganov,

Y. A. Plis, O. N. Shchevelev and Y. A. Usov

Sum Rule Estimates for Heavy and Light Constituent Scattering and Decays 635

Y. N. Srivastava, N. Staffolani, S. Reucroft, J. Swain, A. Widom and

G. Castellani

New NA48 Results on Kaon and Hyperon Decays 639 R. Wanke

Recent and Future Experimental Evidences for Exotic Mesons in Hard Reactions 643

I. V. Anikin, O. V. Teryaev, B. Pire, L. Szymanowski and S. Wallon

Transversity and Collins Asymmetry

A. V. Efremov, K. Goeke and P. Schweitzer

Multiplicity Difference Between Heavy and Light Quark Jets F. Fabbri 
xviii

Measurement of the Pion Polarizabilities at COMPASS 655

A. V. Guskov (on behalf of the COMPASS Collaboration)

New Relations Between DIS Sum Rules . 659

A. L. Kataev

\section{VOLUME II SESSION VII. ELECTROWEAK PHYSICS}

W Width and W, Z Cross Sections at the Tevatron...

$M$. Lancaster (on behalf of the CDF and DØ Collaborations)

$\mathrm{W}$ and $\mathrm{Z}$ Production: Cross-Sections and Asymmetries M. Wielers

Final Results on W Boson Properties from LEP 673 J. D'Hondt

W Mass and Width at the LHC 677 M. Malberti

$W, Z$, and Drell-Yan Production II: Asymmetries 681

$S$. Blessing (on behalf of the $D \varnothing$ and CDF Collaborations)

Charged Current Deep Inelastic Scattering with Polarised $e^{ \pm}$Beams at HERA. . . . 685 A. D. Tapper

$W W$ and $W Z$ Production at the Tevatron 689 E. Lipeles

WW Production, Polarisation and Spin Correlations at LEP 693 P. Azzurri

Numerically Stable Calculations of Radiative Corrections to Bremsstrahlung in $e^{+} e^{-}$Annihilation

S. A. Yost and B. F. L. Ward

Neutral Current DIS with Polarised $e^{ \pm}$at HERA 701 Vladimir Chekelian (Shekelyan)

Measurements of $\tau$ Branching Fractions at B-Factories 705

B. A. Shwartz (on behalf of the BELLE $\mathcal{E}$ BABAR Collaborations)

$t \bar{t}$ Production at the Tevatron: Event Selection and Cross Section Measurement 709 D. C. O'Neil (for the CDF and DØ Collaborations) 
Top Quark Mass Measurements ......................... 713

F. Canelli (for the CDF and DØ Collaborations)

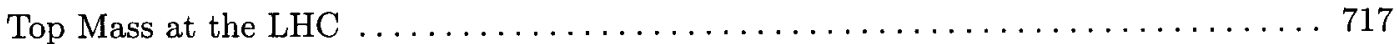

R. Chierici

Top Pair Production III: Testing the Standard Model in Top Quark Decays . . . . . . 721

D. Wicke (on behalf of the CDF and DØ Collaborations)

Combined QCD AND Electro-Weak Fits at HERA .

Y. D. Ri (on behalf of the H1 and ZEUS Collaborations)

Precision Tests of the Standard Model

C. Parkes

Search for Single-Top-Quark Production at the Tevatron

W. Wagner (for the CDF and DØ Collaborations)

Testing the Standard Model in $t \bar{t}$ and Single Top Production at the LHC

A. Onofre

FCNC Top Quark Production and Decay as Analyzers of New Physics

G. Eilam

Weak Radiative Corrections to the Drell-Yan Process for Large Invariant Mass of a Dilepton Pair.

V. A. Zykunov

Search for Low Mass Higgs at the Tevatron

B. Kilminster (on behalf of the CDF and DØ Collaborations)

Search for the Standard Model Higgs Boson at the LHC

A. N. Nikitenko

Studies of Diboson Production and Triple Gauge Boson Couplings at LHC

Z. Zhao (representing CMS and ATLAS Collaborations)

Relativistic Calculation of the Neutron Charge form Factor from

JLAB Deuteron Data.

A. F. Krutov and V. E. Troitsky

Matching Parton Shower and Matrix Elements in QED

G. Balossini, C. M. Carloni Calame, O. Nicrosini, F. Piccinini and

G. Montagna

\section{SESSION VIII. CP VIOLATION, RARE DECAYS, CKM}

New Perspectives on Kaon Physics from KLOE . 773

T. Spadaro 
Recent Results from ISTRA + Experiment $\ldots \ldots \ldots \ldots \ldots \ldots \ldots \ldots \ldots \ldots \ldots 77$ V. A. Duk

Search for Direct CP-Violation in $K^{ \pm} \rightarrow 3 \pi$ Decay by NA48/2 $\ldots \ldots \ldots \ldots \ldots \ldots 78$ S. Balev

$\left|V_{u s}\right|$ from Strange Hadronic Tau Data . . . . . . . . . . . . . . . . . . . . 786

E. Gámiz, M. Jamin, A. Pich, J. Prades and F. Schwab

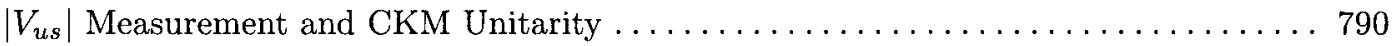
M. Antonelli

Experimental Search for $K_{L}^{0} \rightarrow \pi^{0} \nu \bar{\nu}$ 794

M. Doroshenko (for E391a Collaboration)

Future of Kaon Experiments

A. Ceccucci

Search for $D^{0}-\bar{D}^{0}$ Mixing with the BABAR Experiment

M. G. Wilson (for the BABAR Collaboration)

Cabibbo-Allowed and Doubly Cabibbo Suppressed $D \rightarrow K \pi$ Decays . . . . . . . 807 S. R. Blusk

Search for New Physics in Rare D Decays .......................... 811 S. Fajfer and S. Prelovšek

The $\phi_{1}$ Measurements with Tree-Level Processes at BELLE

O. Tajima (for the BELLE Collaboration)

$C P$ Violation Measurements in $\mathrm{B} \rightarrow$ Charm Decays at $B A B A R \ldots \ldots \ldots \ldots \ldots \ldots . \ldots 19$ K. A. George

Observation of Time-Dependent $C P$-Violation in $B^{0} \rightarrow \eta^{\prime} K^{0}$ Decays at BELLE $\ldots 823$ $K$. Hara (for the BELLE Collaboration)

Measurements of the CKM Angle $\beta$ in Charmless Loop-Dominated $B$ Meson Decays at BABAR

A. Lazzaro (for the BABAR Collaboration)

Observation of Direct $C P$-Violation in $B^{0} \rightarrow \pi^{+} \pi^{-}$Decays with 535 Million

$B \bar{B}$ Pairs

H. Ishino (for the BELLE Collaboration)

Study of CP Violation in $B_{s}$ System

$G$. Borissov (on behalf of the $D \varnothing$ Collaboration)

Measurements of the $C P$ Angle $\gamma$ with the BABAR Experiment G. Marchiori (for the BABAR Collaboration) 
Measurement of Direct CP Asymmetries in Charmless Hadronic B Decays 843

E. Di Marco (for the BABAR Collaboration)

Rare B Decays at CDF

S. M. Farrington

Rare Heavy Flavor Decays at D $\varnothing$

M. G. Strauss

Status of ATLAS Preparations for B-Physics Measurements . . . . . . . . . 855

S. Yu. Sivoklokov (on behalf of the ATLAS Collaboration)

Study of $B_{s}^{0} \rightarrow \mu^{+} \mu^{-}$in CMS

$U$. Langenegger

Searches for Leptonic $B$ Decays with the BABAR Experiment

S. J. Sekula (MIT) (for the BABAR Collaboration)

A Brief Account of $B \rightarrow K^{*} \ell^{+} \ell^{-}$Decay in Soft-Collinear Effective Theory $\ldots \ldots \ldots 867$ A. Ali

Radiative and Electroweak Penguin Processes in Exclusive B Decays . . . . . . . 871

D. Kovalskyi (for the BABAR Collaboration)

Observation of $B_{s}^{0}-\bar{B}_{s}^{0}$ Oscillations and Measurement of $\Delta m_{s}$ in CDF

$S$. Giagu (for the CDF Collaboration)

$B_{s}^{0}$ Mixing at D $\varnothing$ Experiment

T. Moulik and A. Nomerotski (on behalf of DØ Collaboration)

Status of the Unitarity Triangle in the Standard Model

M. Bona, M. Ciuchini, V. Lubicz, E. Franco, G. Martinelli,

L. Silvestrini, F. Parodi, C. Schiavi, M. Pierini, P. Roudeau,

V. Sordini, A. Stocchi and V. Vagnoni

Mixing and CP-Violation in the Littlest Higgs Model with T-Parity . . . . . . . . 888 C. Tarantino

B-Physics Prospects for the LHCb Experiment 892

T. Ruf (for the LHCb Collaboration)

Neutron $\beta$-Decay, Standard Model and Cosmology 896 A. P. Serebrov

\section{SESSION IX. HADRON SPECTROSCOPY \& EXOTICS}

Lattice Approach to Threshold States 903

C. McNeile 
The Experimental Investigation of the Missing $N^{*}$ - and $\Delta$-Resonances

Problem: Current Status and Perspectives . . . . . . . . . . . . . . . . . 907

V. V. Sumachev, V. S. Bekrenev, Yu. A. Beloglazov, E. A. Filimonov,

A. I. Kovalev, N. G. Kozlenko, S. P. Kruglov, A. A. Kulbardis, I. V. Lopatin,

D. V. Novinsky, V. A. Shchedrov, V. Yu. Trautman, I. G. Alekseev,

P. E. Budkovsky, V. P. Kanavets, L. I. Koroleva, B. V. Morozov,

V. M. Nesterov, V. V. Ryltsov, A. D. Sulimov, D. N. Svirida,

N. A. Bazhanov and E. I. Bunyatova (for PNPI-ITEP Collaboration)

Charmed Meson Spectroscopy at BELLE

J. Brodzicka

Study of Recently Observed Charm States and Observation of New States with

the BABAR Experiment .

D. Del Re (for the BABAR Collaboration)

Study of Charm Baryons with the BABAR Experiment

B. Aa. Petersen (representing the BABAR Collaboration)

Latest Results on Orbitally Excited Strange Bottom Mesons with the

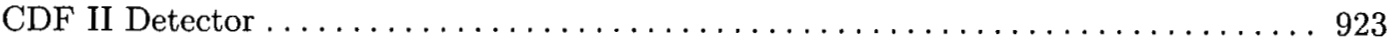

I. V. Gorelov (for the CDF Collaboration)

YXYZX Roads: Charmonium and Beyond $\ldots \ldots \ldots \ldots \ldots \ldots \ldots \ldots \ldots \ldots \ldots$

R. Mussa

Charmonium and Charmonium-Like Mesons at BELLE

G. Majumder (on the behalf of the BELLE Collaboration)

New Results on $X(3872)$ from CDF

M. Kreps (on behalf of the CDF Collaboration)

Search for Bottom Counterparts of $X(3872)$ and $Y(4260)$ via $\pi^{+} \pi^{-} \Upsilon$

G. W. S. Hou

$X(3872)$ in the Coupled-Channel Model

Yu. S. Kalashnikova

Recent Results on $\psi(3770)$ and $\psi(2 S)$ Production and Decays at BES 947

G. Rong (for the BES Collaboration)

Results of Hadronic Cross Section Measurement at VEPP-2M Collider with

CMD-2 and SND Detectors

R. R. Akhmetshin, M. N. Achasov, V. M. Aulchenko, V. Sh. Banzarov,

L. M. Barkov, N. S. Bashtovoy, K. I. Beloborodov, A. V. Berdyugin,

A. E. Bondar, D. V. Bondarev, A. V. Bragin, A. G. Bogdanchikov,

A. V. Bozhenok, A. D. Bukin, D. A. Bukin, T. V. Dimova, V. P. Druzhinin,

S. I. Eidelman, D. A. Epifanov, G. V. Fedotovich, N. I. Gabyshev,

V. B. Golubev, D. A. Gorbachev, A. A. Grebenuk, D. N. Grigoriev, 
F. V. Ignatov, S. V. Karpov, V. F. Kazanin, B. I. Khazin, I. A. Koop,

A. A. Korol, S. V. Koshuba, P. P. Krokovny, A. S. Kuzmin,

I. B. Logashenko, P. A. Lukin, A. P. Lysenko, K. Yu. Mikhailov,

A. I. Milshtein, I. N. Nesterenko, M. A. Nikulin, V. S. Okhapkin,

A. V. Otboev, E. V. Pakhtusova, E. A. Perevedentsev, A. S. Popov,

S. I. Redin, N. I. Root, A. A. Ruban, N. M. Ryskulov, S. I. Serednyakov,

A. G. Shamov, Yu. M. Shatunov, B. A. Shwartz, A. L. Sibidanov,

V. A. Sidorov, Z. K. Silagadze, A. N. Skrinsky, V. P. Smakhtin,

I. G. Snopkov, E. P. Solodov, Yu. A. Tikhonov, A. V. Vasiljev,

Yu. V. Yudin, A. S. Zaitsev and S. G. Zverev

The Lowest Scalar Resonance of QCD

I. Caprini

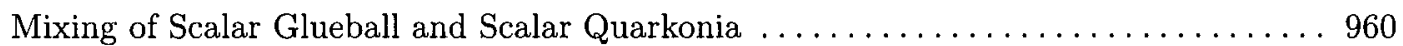
H.- Y. Cheng

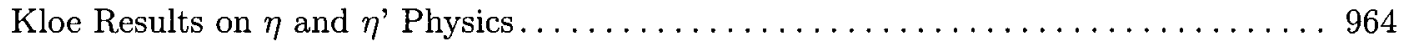

B. Di Micco (for the KLOE Collaboration)

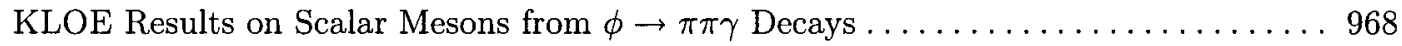

F. Nguyen (for the KLOE Collaboration)

Search for the Exotic $\Theta^{+}$Baryon in Neutrino Interactions in the NOMAD

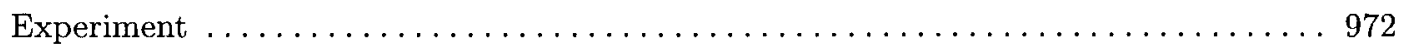

V. Cavasinni (on behalf of the NOMAD Collaboration)

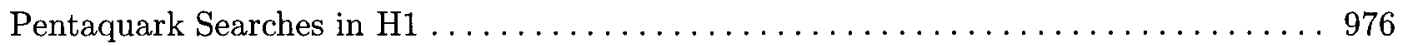

J. E. Olsson (on behalf of the H1 Collaboration)

Charm Spectroscopy and Exotics at ZEUS ....................... 980

L. K. Gladilin (on behalf of the ZEUS Collaboration)

Pseudoscalar-Scalar Transition form Factors in Covariant Light Front Dynamics . . . 984

O. M. A. Leitner, B. El-Bennich, B. Loiseau and J. P. Dedonder

Experimental Study of Resonant Like Structure in the Pion Production Reaction

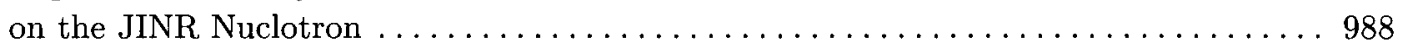

V. A. Krasnov, YU. S. Anisimov, S. Gmuca, M. Janek, E. V. Karpechev,

V. A. Kizka, S. N. Kuznetsov, V. P. Ladygin, A. N. Livanov, A. I. Malakhov,

J. Kliman, V. Matoushek, M. Morkhach, E. B. Plekhanov, N. S. Topil'skaja,

I. Turzo, A. B. Kurepin and T. A. Vasiliev

Study of Narrow Baryon Resonance Decaying into $K_{s}^{0} p$ in $p A$-Interactions at

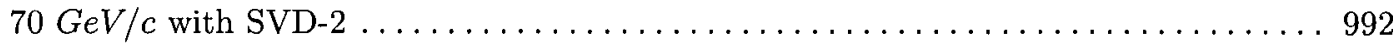

A. Kubarovsky, V. Popov and V. Volkov (for the SVD-2 Collaboration) 


\section{SESSION X. HEAVY QUARK PHYSICS}

Study of the Two-Body Charmless B Decays with the BABAR Experiment . . . . . . . 999 M. Bona (representing the BABAR Collaboration)

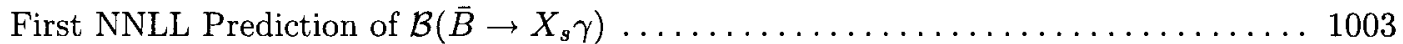

T. Hurth

Study of Multi-Body Charmless $B$ Decays with the BABAR Experiment . . . . . . . 1008

T. E. Latham (representing the BABAR Collaboration)

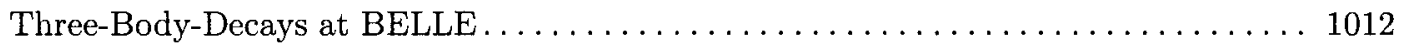

J. Schümann (for the BELLE Collaboration)

Recent Developments in Soft-Collinear Effective Theory. . . . . . . . . . . . 1016 Th. Feldmann

Study of the Decays of Charm Mesons with the BABAR Experiment . . . . . . . 1020 $M$. Bondioli (representing the BABAR Collaboration)

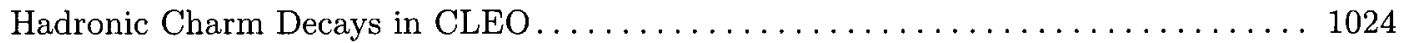
S. R. Blusk

Penguin Amplitudes in Hadronic B Decays: NLO Spectator Scattering . . . . . . . 1028 S. Jäger

Measurement of $D_{s}^{+} \rightarrow \ell^{+} \nu$ and the Decay Constant $f_{D_{s}} \ldots \ldots \ldots \ldots \ldots \ldots \ldots \ldots 1032$ S. Stone

Radiative $B$ Decays at BELLE 1036

A. Limosani (for the BELLE Collaboration)

The Isgur-Wise Function in the BPS Limit . . . . . . . . . . . . . . . . 1040

F. Jugeau, A. Le Yaouanc, L. Oliver and J.-C. Raynal

Recent Measurements of $\left|V_{u b}\right|$ at $B A B A R$ 1044

R. S. Dubitzky (for the BABAR Collaboration)

Exclusive $B \rightarrow X_{u} \ell \nu$ Decays at BELLE and Extraction of $\left|V_{u b}\right| \ldots \ldots \ldots \ldots \ldots \ldots 1048$ C. Schwanda (for the BELLE Collaboration)

Inclusive $B \rightarrow X_{c} l \nu$ Decay Spectra at BELLE and the Determination of $\left|V_{c b}\right| \ldots \ldots 1053$ $P$. Urquijo (for the BELLE Collaboration)

$b \rightarrow c \ell \nu_{\ell}$ Decays and Measurements of $\left|V_{c b}\right|$ with the BABAR Detector 1058 D. L. Pegna (representing the BABAR Collaboration)

Recent Top Quark Results From D $\varnothing$ 1062

S. Anderson 
Relativistic Description of Semileptonic Decays of Heavy Baryons . . . . . . . . . 1066 D. Ebert, R. N. Faustov and V. O. Galkin

B Hadron Properties with DØ Detector 1070 A. Nomerotski (on behalf of DØ Collaboration)

Study of B Decays to Open Charm Final States with the BABAR Experiment . . . . 1075 G. Calderini (on behalf of the BABAR Collaboration)

$b \rightarrow c$ Hadronic B Decays at BELLE

S. C. Blyth (for the BELLE Collaboration)

Double Charm Production in $e^{+} e^{-}$Annihilation 1084 A. K. Likhoded

Hot Topics from BELLE 1087

A. Drutskoy (for the BELLE Collaboration)

Masses and Decay Constants of $B_{q}$ Mesons in QCD String Approach 1091 A. M. Badalian, Yu. A. Simonov and B. L. G. Bakker

Recent Results of Charmonium Decays at BESII 1095 R.-G. Ping (for the BES Collaboration)

\section{SESSION XI. BEYOND THE STANDARD MODEL}

Results of Beyond the Standard Model Higgs Searches from the LEP Experiments . . 1101 A. Ludwig (on behalf of the four LEP Collaborations)

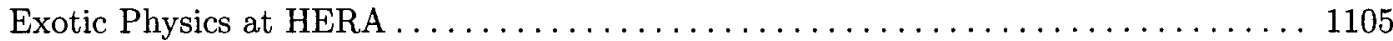

G. Brandt (on behalf of the H1 and ZEUS Collaborations)

Non-Standard Model Higgs Searches at the Tevatron . . . . . . . . . . . . . . 1109 A. Hocker (for the CDF and DØ Collaborations)

Phenomenology of Littlest Higgs Model with T-parity: Including Effects of T-odd Fermions.

A. Belyaev, C.-R. Chen, K. Tobe and C.-P. Yuan

SUSY Searches at HERA

L. Bellagamba

Search for SUSY at the Tevatron

T. Hebbeker (on behalf of the CDF and $D \varnothing$ Collaborations)

Collider Signatures of $\mathrm{E}_{6} \mathrm{SSM}$

S. F. King, S. Moretti and R. Nevzorov 
xxvi

LHC SUSY Discovery Potential . . . . . . . . . . . . . . . . . . . . . . . . . . 1129

$V$. Zhukov (on behalf of the CMS and ATLAS Collaborations)

Searches for SUSY and Extra Dimensions at LEP $\ldots \ldots \ldots \ldots \ldots \ldots \ldots \ldots \ldots \ldots$

$K$. Klein (on behalf of the LEP Collaborations)

Searches for Excited and New Leptons and Quarks at the Tevatron............ 1137

H. B. Greenlee (for the CDF and DØ Collaborations)

Search for Extra Dimensions with the ATLAS and CMS Detectors at the LHC . . . 1141

S. Shmatov (for the ATLAS and CMS Collaborations)

New Physics Searches at LEP

F. Veloso

Events with Isolated Leptons and Missing Transverse Momentum at HERA

C. Diaconu (on behalf of the H1 and ZEUS Collaborations)

Searches for Leptoquarks and Contact Interactions at HERA

J. Sztuk-Dambietz (on behalf of the H1 and ZEUS Collaborations)

Some Searches for New Physics with the DØ Detector

E. Kajfasz (for the DØ Collaboration)

Search for New Physics with Photons at CDF

A. Pronko (for the CDF Collaboration)

Beyond the Standard Model Searches at the LHC ...

S. Willocq (representing the ATLAS and CMS Collaborations)

Mass Ambiguities in Cascade Decays .

B. K. Gjelsten, D. J. Miller, P. Osland and A. R. Raklev

Lepton Flavor Violation Searches at B-Factories

K. Hayasaka

Probing New Physics in $B_{s}$ and $D$ Mixings, and $A_{\mathrm{CP}}\left(B^{+} \rightarrow J / \psi K^{+}\right)$

G. W. S. Hou

Testing New Physics with Unitarity Triangle Fits

M. Bona, M. Ciuchini, V. Lubicz, E. Franco, G. Martinelli, L. Silvestrini,

F. Parodi, C. Schiavi, M. Pierini, P. Roudeau, V. Sordini, A. Stocchi and

V. Vagnoni

\section{SESSION XII. GRID DISTRIBUTED ANALYSIS IN HIGH ENERGY PHYSICS}

ALICE Physics Analysis Using the Grid 1189

I. Belikov (for the ALICE Collaboration) 
The First Year of LHC Physics Analysis Using the Grid: Prospects from CMS . . . . 1193

I. Fisk (for the CMS Collaboration)

Grid User Interfaces for $\mathrm{LHC} \ldots \ldots \ldots \ldots \ldots \ldots \ldots \ldots \ldots \ldots \ldots \ldots \ldots \ldots \ldots \ldots$

J. Andreeva

Archiving of Particle Physics Data and Results for Long-Term Access and Use . . . . 1201

J. Yeomans

\section{SESSION XIII. R \& D FOR FUTURE ACCELERATORS \& DETECTORS}

Muon Cooling and Future Muon Facilities ....................... 1207

D. M. Kaplan

Simulation of Mice Using G4MICE

L. Howlett and C. Rogers

A High Intensity Linear $e^{+} e^{-}$Collider Facility at Low Energy

A. Schöning

\section{SESSION XIV. MATHEMATICAL ASPECTS OF QFT \& STRING THEORY}

Decay of Metastable Vacuum in Liouville Gravity . . . . . . . . . . . . . . . . . . 1223

A. Zamolodchikov and Al. Zamolodchikov

Duality in Supersymmetric Yang-Mills and the Quantum Hall Effect

B. P. Dolan

Resummed Quantum Gravity .

B. F. L. Ward

Cosmology from Cubic Superstring Field Theory Model

A. S. Koshelev

Noncommutative Quantum Field Theory: Twisted Poincaré Symmetry and

Its Implications

A. Tureanu

Black Ring Solutions in 5D Heterotic String Theory: A Full Field Configuration . . . 1246

A. Herrera-Aguilar and H. R. Marquez-Falcon

Axiomatic Approach in Noncommutative Quantum Field Theory

Yu. S. Vernov

Zero-Norm States and Superstringy Symmetries

J.-C. Lee

List of Posters 